\title{
Construção e Validação da Escala de Teorias Implícitas de Inteligência Emocional (TIIE) no Contexto Escolar
}

\author{
Development and Validation of the Implicit Theories of Emotional Intelligence Scale \\ (ITEI) in the Academic Context
}

\author{
Ana Costa ${ }^{1}$ e Luísa Faria ${ }^{2}$
}

\begin{abstract}
Resumo
A literatura tem demonstrado que indivíduos com teorias implícitas dinâmicas apresentam melhores resultados escolares e profissionais, níveis superiores de bem-estar e experiências emocionais mais positivas. Ora, a avaliação das teorias implícitas nos domínios emocionais é, ainda, escassa. Assim, pretende-se avaliar as qualidades psicométricas de uma nova escala de teorias implícitas de inteligência emocional (TIIE), numa amostra de 612 adolescentes. A análise fatorial exploratória da escala evidenciou uma estrutura de dois fatores - dinâmico e estático - que explicam $54.2 \%$ da variância total dos resultados. A análise fatorial confirmatória comprovou o modelo bifatorial, sendo que a escala apresentou invariância de género. Os coeficientes alfa de Cronbach e half-split apresentaram-se superiores a .90. A TIIE apresentou validade discriminante quanto a medidas afins, como medidas de teorias implícitas de inteligência e de emoções face à escola. Globalmente, os resultados sugerem que a TIIE se revelou psicometricamente válida para avaliar as teorias implícitas da inteligência emocional.
\end{abstract}

Palavras-chave: teorias implícitas, inteligência emocional, qualidades psicométricas, contexto escolar

\begin{abstract}
Literature has shown that incremental theorists have greater scholastic and professional results, higher wellbeing and more positive emotional experiences. Nonetheless, measurement in the implicit theories of emotional-related domains is still scarce. Therefore, this paper examined the properties of a newly developed implicit theories of emotional intelligence scale (ITEI) among a sample of 612 adolescents. The exploratory factor analysis evidenced the scale's two-factor structure - incremental and entity - with $54.2 \%$ of total explained variance. Confirmatory factor analysis established the bifactorial model, and ITEI presented invariant measurement across gender. Cronbach's alpha and half-split coefficients were above .90. ITEI presented discriminant validity with related measures, such as the implicit theories of intelligence and emotions towards school. Globally, the results suggest that ITEI is a psychometrically sound measure to assess implicit theories of emotional intelligence.
\end{abstract}

Keywords: implicit theories, emotional intelligence, psychometric properties, academic context

\footnotetext{
${ }^{1}$ Bolseira de Pós-Doutoramento em Psicologia da FCT na Faculdade de Psicologia e de Ciências da Educação da Universidade do Porto, Porto, Portugal. (ref. ${ }^{a}$ SFRH/BPD/117479/2016). E-mail: ana.fontescosta@ gmail.com

${ }^{2}$ Professora Associada com Agregação da Faculdade de Psicologia e de Ciências da Educação da Universidade do Porto, Porto, Portugal. E-mail:Ifaria@fpce.up.pt

Revista Iberoamericana de Diagnóstico y Evaluación - e Avaliação Psicológica. RIDEP · N54 · Vol.1 · 57-68 · 2020 ISSN: 1135-3848 print /2183-6051online
} 


\section{Introdução}

As teorias implícitas funcionam como crenças nucleares que afetam o comportamento quotidiano dos indivíduos e as perceções acerca de si próprios e dos outros (Chiu, Hong, \& Dweck, 1997; Dweck, 2012a, 2012b). A investigação tem indicado que os indivíduos podem diferir significativamente nas suas crenças sobre atributos humanos fundamentais. Os indivíduos que detêm crenças mais dinâmicas sobre atributos particulares têm evidenciado resultados mais positivos em diferentes domínios da vida (por exemplo, realização académica e profissional, bem-estar, experiências emocionais e relacionamentos positivos; Dweck, 2012a). Na verdade, a investigação no domínio das teorias implícitas sobre a emoção está no seu início, sendo particularmente escassa no quadro da inteligência emocional. $O$ presente trabalho pretende dar um contributo neste domínio, através da proposta de uma medida válida de teorias implícitas de inteligência emocional.

\section{Teorias Implícitas}

Os indivíduos podem apresentar diferentes sistemas de significado fundados na sua perspetiva acerca da flexibilidade ou inflexibilidade de atributos importantes como a inteligência, a emoção, as habilidades sociais e interpessoais ou a personalidade (Dweck, 2012a): indivíduos que apresentam teorias estáticas acreditam que essas características são predeterminadas, relativamente fixas e dificilmente mutáveis, enquanto que os que apresentam teorias dinâmicas consideram-nas como traços e qualidades flexíveis, que podem ser transformadas, com trabalho e esforço, através da aprendizagem e do investimento (Dweck \& Legget, 1988). No entanto, as teorias implícitas são específicas de cada domínio e os indivíduos podem ter teorias independentes sobre diferentes atributos (por exemplo, inteligência, moralidade ou personalidade; Chiu et al., 1997; Dweck, Chiu, \& Hong, 1995).

Num dos domínios mais estudados das teorias implícitas, a inteligência, a investigação indicou que os dinâmicos perspetivam o esforço como um requisito fundamental para desenvolver a capacidade/inteligência, o que os leva a ser mais persistentes e estratégicos e a manifestar comportamentos autorregulados adaptativos quando enfrentam desafios e dificuldades (Blackwell, Trzesniewski, \& Dweck, 2007; Burnette, O'Boyle, VanEpps, Pollack, \& Finkel, 2013). Considerando o desempenho escolar, os estudantes que possuem teorias dinâmicas de inteligência tendem a apresentar vantagem em comparação com os estáticos, uma vez que as pesquisas demonstraram que alcançam resultados significativamente mais elevados (ver revisão, Costa \& Faria, 2018; Faria, 2008).

Mais recentemente, estudos no domínio têm explorado as teorias implícitas de atributos relacionados com a emoção. Em particular, a literatura demonstrou que as teorias implícitas da emoção podem ter efeitos na perceção, motivação e comportamento dos indivíduos. Na verdade, os indivíduos com teorias mais estáticas das emoções relataram emoções negativas mais intensas, depressão, bem-estar psicológico reduzido e autoeficácia inferior na regulação emocional (Kappes \& Schikowski, 2013; Tamir, Srivastava, \& Gross, 2007). Por outro lado, os indivíduos dinâmicos experimentaram mais emoções positivas, receberam maior apoio social, usaram estratégias orientadas para a mestria com mais frequência e desenvolveram melhores expectativas de sucesso (De Castella et al, 2013; Kappes \& Schikowski, 2013; Romero, Master, Paunesku, Dweck, \& Gross, 2014; Tamir et al., 2007).

Mais ainda, vários estudos correlacionaram positivamente teorias implícitas mais dinâmicas da empatia com comportamentos empáticos: os indivíduos dinâmicos foram mais empáticos em contextos desafiantes, desenvolveram esforços para responder de forma mais empática a pessoas com perspetivas sociopolíticas conflituantes, estavam mais predispostos a ouvir outras histórias pessoais com conteúdo emocional, a ajudar pacientes em interação face-a-face e a desenvolver a sua própria empatia (Schumann, Zaki, \& Dweck, 2014).

\section{Inteligência Emocional}

A investigação no domínio da Inteligência Emocional (IE) prosperou significativamente desde que Salovey e Mayer apresentaram o conceito em 1990, sendo que, atualmente, existe 
um amplo consenso sobre o facto da IE ser um fator importante na explicação do sucesso, desempenho e realização dos indivíduos (Brackett, Rivers, \& Salovey, 2011; FernándezBerrocal \& Extremera, 2006; Salessi \& Omar, 2016; Van Rooy \& Viswesvaran, 2004). Em particular, no contexto académico, a literatura mostrou que a IE reforça o desenvolvimento cognitivo e social das crianças (Mavroveli \& Sánchez-Ruiz, 2011; Mestre, Guil, Lopes, Salovey, \& Gil-Olarte, 2006) e prediz o desempenho académico dos alunos (Billings, Downey, Lomas, Lloyd, \& Stough, 2014; Costa \& Faria, 2015).

Os modelos teóricos de IE mais comummente aceites pela comunidade científica são o modelo de habilidade e o modelo de traço (Mayer, Roberts, \& Barsade, 2008). Conceptualizada como uma habilidade, a IE integra um conjunto de competências inter-relacionadas que permitem perceber e expressar emoção, aceder e/ou gerar sentimentos que facilitam o pensamento, compreender a emoção e regular as emoções no próprio e nos outros (Mayer \& Salovey, 1997), pertencendo, assim, ao domínio da capacidade cognitiva e sendo avaliada através de medidas objetivas de desempenho. O Teste de Inteligência Emocional Mayer-Salovey-Caruso - MSCEIT constitui um teste clássico de IE, sendo um dos mais utilizados no domínio, com qualidades psicométricas aceitáveis (Teixeira, \& Araújo, 2018). O modelo de traço de IE é conceptualizado como uma constelação de perceções emocionais que, neste caso, devem ser avaliadas através de questionários e escalas de autoavaliação (Petrides, Pita, \& Kokkinaki, 2007).

Num estudo precursor, desenvolvido por Cabello e Fernández-Berrocal (2015) sobre as teorias implícitas da inteligência emocional, os autores estabeleceram que os indivíduos com conceções mais dinâmicas da inteligência emocional e da emoção apresentavam níveis mais elevados de IE, avaliada por meio de uma medida de desempenho. De facto, os resultados corroboram que os indivíduos dinâmicos tendem a usar mais frequentemente estratégias eficazes de reavaliação cognitiva e orientadas para a mestria $\mathrm{e}$, consequentemente, desenvolvem habilidades emocionais mais positivas (Cabello \& FernándezBerrocal, 2015).
Não há evidência empírica clara de que o género possa influenciar as teorias implícitas dos indivíduos. A literatura tem vindo a apresentar resultados contrastantes: embora haja estudos que demonstraram que o género feminino pode ter uma perspetiva mais estática da inteligência (Dweck, 1999; Faria, 2008; Pepi, Faria, \& Alesi, 2006), estudos mais recentes não observaram quaisquer diferenças entre os grupos (Burnette et al., 2013; Costa \& Faria, 2018). Na investigação sobre as teorias implícitas da inteligência emocional, os resultados demonstraram diferenças de género, favorecendo as mulheres, que apresentam conceções mais dinâmicas (Cabello \& Fernández-Berrocal, 2015).

\section{$O$ presente estudo}

No contexto português, aparentemente, nenhum outro estudo explorou as teorias implícitas da inteligência emocional ou desenvolveu uma medida válida para a sua avaliação. Neste sentido, pretende-se explorar este constructo, desenvolvendo e adaptando uma Escala de Teorias Implícitas de Inteligência Emocional (TIIE), avaliando as suas qualidades psicométricas numa amostra de adolescentes no contexto do ensino secundário.

$\mathrm{O}$ ensino secundário é um ciclo particularmente desafiador, pois implica a entrada num ambiente académico mais competitivo e é determinante não só na realização académica como na realização profissional futura. Tendo em consideração que a investigação tem destacado que, em particular, em ciclos de ensino mais desafiadores, as teorias implícitas tendem a evidenciar-se e a influenciar o comportamento e o desempenho dos indivíduos (Blackwell et al., 2007), neste estudo foi incluída uma amostra de alunos do ensino secundário, do $100^{\circ}$ ano, para explorar a fiabilidade e a validade desta escala neste importante contexto de transição.

Assim, quatro objetivos específicos foram propostos: a) analisar a estrutura da TIIE através da análise fatorial exploratória (AFE) e da análise fatorial confirmatória (AFC); b) testar a invariância da medida da TIIE quanto ao género; c) examinar a consistência interna da TIIE; e d) analisar a validade discriminante da TIIE com uma medida de teorias implícitas e uma medida das emoções dos alunos face à escola. A partir da 
investigação anterior no domínio das teorias implícitas (De Castella \& Byrne, 2015; Dupeyrat \& Mariné, 2005), é esperado que a TIIE evidencie uma estrutura bifatorial, que o instrumento suporte a invariância da medida quanto ao género e apresente boa consistência interna. Além disso, espera-se que a TIIE evidencie boa fiabilidade. É também esperado que a escala apresente validade discriminante, ao estabelecer correlações moderadas positivas com as teorias implícitas da escala de inteligência (escala total e subescala dinâmica) e negativas com a subescala estática e, com menor magnitude, correlações positivas com a medida das emoções dos alunos face à escola (escala total e subescala de emoções positivas) e negativas com a subescala de emoções negativas dos alunos.

\section{Método}

\section{Participantes}

A amostra compreende um total de 612 estudantes do $10 .^{\circ}$ ano, com idades compreendidas entre os 14 e os 19 anos $(\mathrm{M}=15.6$; $\mathrm{DP}=.75)$. Cerca de $53.8 \%$ eram raparigas e, globalmente, os estudantes frequentavam diferentes áreas $(63.4 \%$ Científicos e Tecnológicos, 25.7\% Línguas e Humanidades e $10.9 \%$ outros). A amostra inicial foi distribuída aleatoriamente em duas subamostras: assim, a análise fatorial exploratória (AFE) foi realizada na subamostra 1, com 306 estudantes (53.9\% raparigas), com média de idades de 15.6 anos (DP=.76); já a análise fatorial confirmatória (AFC) foi realizada no subgrupo 2 , com 306 estudantes (53.6\% raparigas), com média de idades de 15.6 anos $(\mathrm{DP}=.74)$. Não foram encontradas diferenças estatisticamente significativas entre as subamostras, considerando o género e a idade (género, $\chi^{2}=.01, p=.06$; idade, $t$ $(607)=-.078, p=.94)$.

\section{Medidas}

\section{Teorias Implícitas de Inteligência Emocional}

As teorias implícitas de inteligência emocional dos adolescentes foram avaliadas com a Escala de Teorias Implícitas de Inteligência Emocional (TIIE) desenvolvida neste estudo. A TIIE foi baseada na teoria original de Dweck de teorias implícitas da inteligência (1999), em particular na recente versão da escala de teorias de inteligência aplicada ao self (De Castella \& Byrne, 2015), ou seja, os itens refletem a afirmação da primeira pessoa quanto à flexibilidade da inteligência emocional. Os itens dinâmicos e estáticos foram desenvolvidos por especialistas no domínio e testados em momentos de reflexão falada com estudantes com características semelhantes aos da amostra do estudo principal, para corrigir possíveis imprecisões linguísticas e evitar ambiguidades ou erros gramaticais. A versão final da TIIE é composta por 12 itens (seis dinâmicos e seis estáticos) avaliados por escala de resposta de tipo Likert, de 6 pontos, de 1 (concordo totalmente) a 6 (discordo totalmente). Os itens dinâmicos foram classificados de forma invertida, de modo a que a pontuação média refletisse a medida das conceções dinâmicas de IE dos alunos.

\section{Teorias Implícitas de Inteligência}

As teorias implícitas de inteligência dos estudantes foram avaliadas por uma versão revista da Escala de Conceções Pessoais de Inteligência (ECPI) de Faria (2008), fundada na teoria das conceções pessoais da inteligência de Dweck (1999). A escala compreende 12 itens (seis dinâmicos e seis estáticos), avaliados por escala de resposta de tipo Likert, de 6 pontos, de 1 (concordo totalmente) a 6 (discordo totalmente). A pontuação média da escala indicava a medida das perceções dinâmicas sobre a inteligência, isto é, maior pontuação correspondia a maior dinamismo. Neste estudo, a escala apresentou uma boa consistência interna $(\alpha=.89)$.

\section{Emoções dos Alunos face à Escola}

A escala de Emoções dos Alunos face à Escola é uma medida de autorrelato baseada no Questionário de Emoções Académicas de Pekrun e colaboradores (Pekrun, Goetz, Frenzel, Barchfeld, \& Perry, 2011). A escala avalia as emoções dos alunos face à escola e compreende 9 itens, que descrevem emoções positivas (4) e negativas (5), avaliadas por escala de resposta de frequência, de 6 pontos (de $1=$ nunca a $6=$ sempre). Neste estudo, a escala apresentou uma consistência interna satisfatória $(\alpha=.68)$. 


\section{Procedimento}

As escolas com ciclo de ensino secundário que aceitaram colaborar, indicaram as turmas a incluir no estudo, de acordo com a sua disponibilidade. Os participantes preencheram os instrumentos, individualmente, em administrações coletivas, em sala de aula e em horário escolar. Antes da administração, os alunos foram informados sobre os objetivos do estudo e a confidencialidade e $\mathrm{o}$ anonimato foram garantidos. Os participantes menores de idade apenas foram incluídos no estudo com a autorização dos seus Encarregados de Educação ou responsáveis legais, através de consentimento informado. A colaboração dos estudantes foi voluntária e o estudo não ofereceu qualquer compensação económica. Este estudo foi aprovado pela Comissão Nacional de Proteção Dados e pela Direção-Geral da Educação (Inquéritos em meio escolar) e obteve parecer favorável da Comissão de Ética da Faculdade de Psicologia e de Ciências da Educação.

\section{Análise de dados}

A estrutura fatorial da escala TIIE foi explorada através de análise fatorial exploratória (AFE) na subamostra 1 . O método de extração foi a máxima verossimilhança (MV), assumindo a distribuição normal dos dados e a rotação oblíqua (direct oblimin) foi aplicada, devido à suposta correlação dos fatores subjacentes. O teste de Kaiser-Meyer-Olkin e o teste de esfericidade de Bartlett foram utilizados para avaliar as propriedades da matriz de correlação entre itens. O número ideal de fatores foi baseado nos valores próprios originais (valores dos dados brutos $>1$; critério de Kaiser), inspeção do scree plot, percentagem da variância explicada e subsequente comparação com os resultados da Análise Fatorial Paralela. A análise Paralela foi executada no SPSS IBM (v. 25.0) usando o módulo rawpar.sps desenvolvido por O'Connor (2000). Usando uma abordagem de análise fatorial comum, 2000 conjuntos de dados foram criados com base nos dados brutos. Este método, compara os valores próprios obtidos a partir da base de dados, com os valores próprios médios e com os valores próprios do percentil 95, produzidos pela simulação de Monte Carlo.
Na subamostra 2, a amostra de validação, foi realizada a análise fatorial confirmatória (AFC) com estimador de MV para confirmar o ajustamento do modelo fatorial, previamente obtido na AFE. Os índices Qui-quadrado $\left(\chi^{2}\right)$, Confirmatory Factor Index (CFI), Tucker-Lewis Index (TLI), Root Mean Square Error of Approximation (RMSEA) (e IC 90\%) e Standardized Root Mean Square Residual (SRMR) foram utilizados (Hu, \& Bentler, 1999). Como sugerido por Hu e Bentler (1999), o $\chi^{2}$ relativo $\left(\chi^{2} / d f\right)$ deve ser significativo e $\leq 2$, RMSEA e SRMR devem ser .08 ou inferiores para considerar o modelo adequado e CFI e TLI devem ser .95 ou superiores, embora valores acima de .90 sejam considerados adequados. Além disso, explorou-se a invariância da medida entre géneros, através da realização de uma análise de comparação multigrupo utilizando modelos de equações estruturais: $\mathrm{O}$ modelo de invariância configuracional avalia se a estrutura fatorial é invariante entre os grupos; o modelo de invariância métrica inspeciona se as cargas fatoriais dos itens são invariantes entre os grupos; o modelo de invariância escalar explora se o intercepto dos itens são invariantes entre os grupos: $\Delta$ CFI $\leq .01$ e $\Delta$ RMSEA $\leq .015$ entre os níveis métrico e escalar indicam a invariância da escala quanto ao género (Chen, 2007; Cheung \& Rensvold, 2002). Foi avaliada a consistência interna (coeficiente alfa de Cronbach, coeficiente Splithalf e correlação item-escala) no modelo fatorial. A validade discriminante foi analisada entre o score total da escala TIIE e as suas subescalas e TIIE e a medida de Emoções dos alunos face à Escola. Os softwares estatísticos utilizados foram o SPSS IBM Statistics 25.0 e o AMOS 25.0.

\section{Resultados}

\section{Análise Descritiva}

Os valores de assimetria e de curtose de todas as variáveis foram considerados dentro dos limites da normalidade univariada $(<|1|$, respetivamente). As médias dos itens variaram entre $3.98(\mathrm{DP}=1.34)$ a 5.14 ( $\mathrm{DP}=.90)$. Foram identificados 37 casos como outliers multivariados (distância de Mahalanobis com $p<.001)$ e eliminados das análises subsequentes. Nenhum método de imputação de omissões foi utilizado. 


\section{Estrutura fatorial da TIIE}

\section{Análise Fatorial Exploratória (AFE)}

O índice KMO (.91) e o teste de esfericidade de Bartlett $\left(\chi^{2}=1849.87, p<.001\right)$ indicaram a adequabilidade da amostra para a realização da AFE. Consequentemente, realizou-se uma AFE com estimativa de máxima verossimilhança e rotação direct oblimin ( $\mathrm{n}_{\text {subgrupo }} 1=289$ ). Com base nos resultados da AFE (Quadro 1) e inspeção do scree plot (cf. Fig. 1), dois fatores (com valores próprios acima de 1.0) foram obtidos do conjunto de dados aleatórios (cf. Quadro 1). Esses dois fatores explicaram $54.2 \%$ da variância total, que foi considerado como sendo um valor adequado

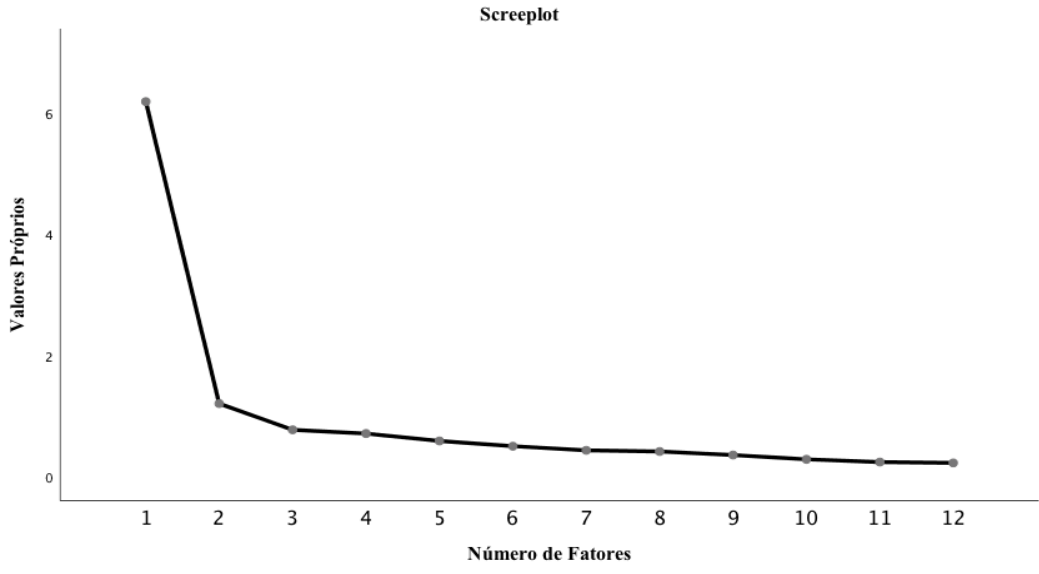

Figura 1. Scree plot na subamostra da AFE $(\mathrm{n}=289)$

Quadro 1. Matriz de componentes após rotação oblíqua (direct oblimin), comunalidades $\left(\mathrm{h}^{2}\right)$, valores próprios e variância explicada

\begin{tabular}{|c|c|c|c|c|}
\hline & Item & Fator I estático & Fator II dinâmico & $h^{2}$ \\
\hline 5. & $\begin{array}{l}\text { Na verdade, acho que não consigo mudar o nível } \\
\text { de inteligência emocional com que nasci. }\end{array}$ & .84 & .58 & .70 \\
\hline 9. & $\begin{array}{l}\text { Por mais que eu me esforce, nunca conseguirei } \\
\text { aumentar a minha inteligência emocional. }\end{array}$ & .83 & .59 & .69 \\
\hline 7. & $\begin{array}{l}\text { Pessoalmente, não posso fazer muito para } \\
\text { aumentar a minha inteligência emocional. }\end{array}$ & .82 & .71 & .69 \\
\hline 4. & $\begin{array}{l}\text { A minha inteligência emocional é uma } \\
\text { característica pessoal que, por mim mesmo/a, não } \\
\text { consigo mudar muito. }\end{array}$ & .76 & .56 & .58 \\
\hline 2. & $\begin{array}{l}\text { Posso ter experiências novas, mas não tenho } \\
\text { capacidade para mudar a minha inteligência } \\
\text { emocional de base. }\end{array}$ & .68 & .51 & .46 \\
\hline 12. & $\begin{array}{l}\text { Não sou tão inteligente emocionalmente quanto } \\
\text { queria, mas não posso fazer nada para mudar } \\
\text { isso. }\end{array}$ & .61 & .48 & .38 \\
\hline 8. & $\begin{array}{l}\text { Eu acredito que tenho a capacidade para mudar } \\
\text { consideravelmente o meu nível de inteligência } \\
\text { emocional atual, ao longo do tempo. }\end{array}$ & .61 & .77 & .60 \\
\hline 3. & $\begin{array}{l}\text { Com tempo e esforço, eu penso que poderei } \\
\text { mudar bastante o meu nível de inteligência } \\
\text { emocional. }\end{array}$ & .56 & .77 & .59 \\
\hline 11. & $\begin{array}{l}\text { Independentemente do meu nível de inteligência } \\
\text { emocional atual, eu posso sempre mudar bastante } \\
\text { este tipo de inteligência. }\end{array}$ & .48 & .72 & .53 \\
\hline 1. & $\begin{array}{l}\text { Com esforço, eu consigo mudar bastante a minha } \\
\text { inteligência emocional. }\end{array}$ & .53 & .69 & .48 \\
\hline 6. & $\begin{array}{l}\text { Os desafios e as dificuldades que enfrento } \\
\text { ajudam-me a desenvolver o meu nível de } \\
\text { inteligência emocional. }\end{array}$ & .55 & .66 & .44 \\
\hline 10. & $\begin{array}{l}\text { Sempre que eu aprendo com experiências novas a } \\
\text { minha inteligência emocional aumenta. }\end{array}$ & .45 & .61 & .37 \\
\hline & Valor Próprio & 6.19 & 1.21 & \\
\hline & \% Variância Explicada & 47.79 & 6.45 & \\
\hline & Soma dos quadrados dos pesos fatoriais & 5.74 & .77 & \\
\hline
\end{tabular}


Quadro 2. Resultados da Análise Fatorial Paralela

\begin{tabular}{lccc}
\hline Fator & $\begin{array}{c}\text { Valor Próprio } \\
\text { dados }\end{array}$ & $\begin{array}{c}\text { Média Valor Próprio } \\
\text { Simulação Monte Carlo }\end{array}$ & \begin{tabular}{c} 
Vercentil 95 Simulação Monte Carlo \\
\hline 1
\end{tabular} S.691782 \\
2 & .748702 & .397419 & .494294 \\
3 & .259372 & .299588 & .377343 \\
4 & .221760 & .222970 & .284754 \\
5 & .094815 & .158796 & .211757 \\
6 & .016040 & .099648 & .144999 \\
7 & .004198 & .046986 & .088561 \\
8 & -.072697 & -.002407 & .037139 \\
9 & -.086014 & -.052119 & -.016284 \\
10 & -.158686 & -.099016 & -.064914 \\
11 & -.178544 & -.147633 & -.112496 \\
12 & -.213410 & -.199131 & -.160556 \\
\hline
\end{tabular}

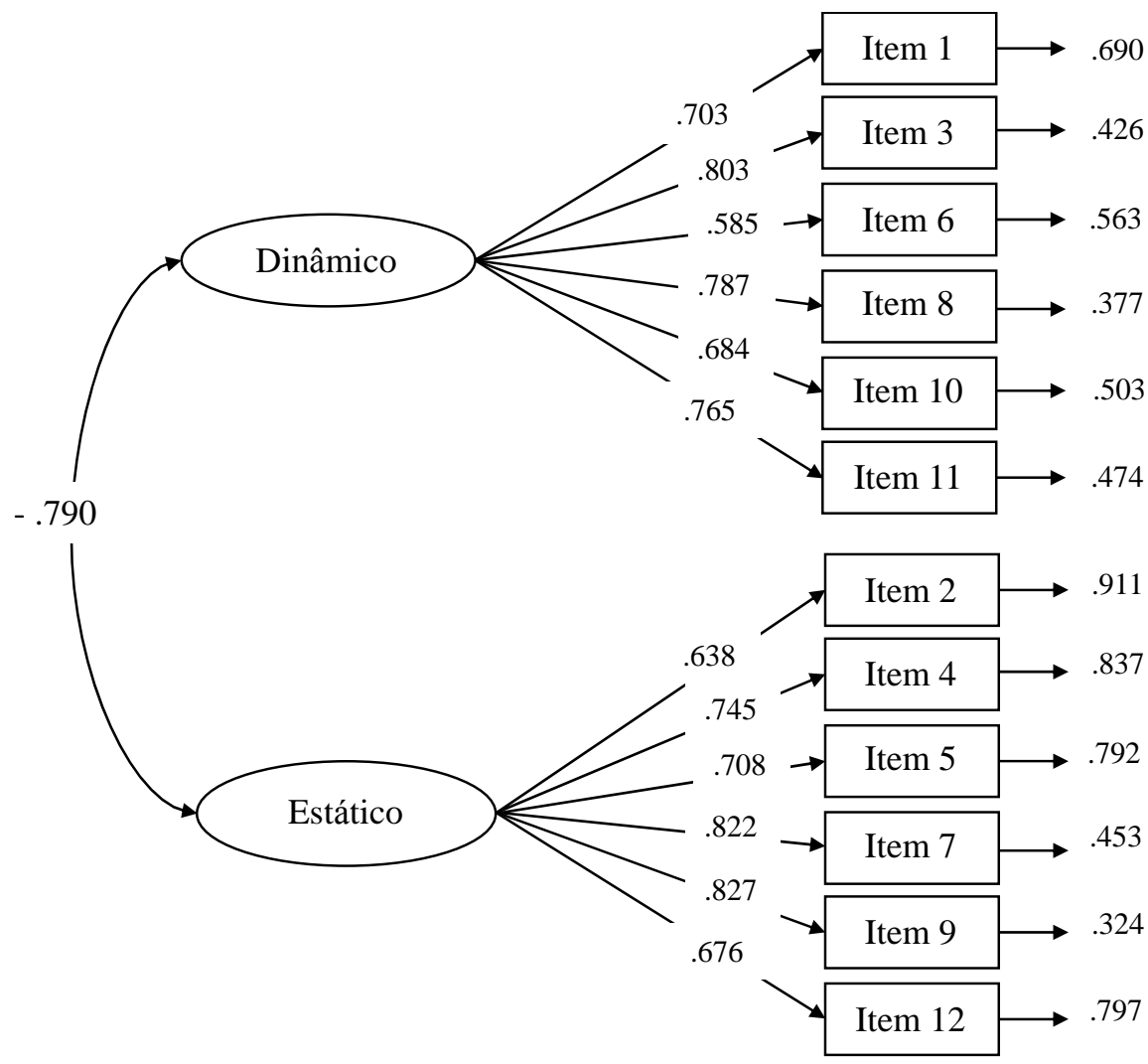

Figura 2. Modelo bifatorial da AFC da TIIE na amostra de validação $(\mathrm{n}=286)$

de variância total explicada. O Quadro 1 indica os itens da escala TIIE e respetivas cargas fatoriais e comunalidades. Os critérios para cargas fatoriais adequadas foram considerados acima de .30 e para comunalidades acima de .40 .

Os resultados da análise paralela indicaram que, dos dados brutos produzidas pela simulação de Monte Carlo, emergiram 3 fatores que se situaram acima das estimativas do percentil 95 (cf. Quadro 2). No entanto, o terceiro fator identificado foi apenas marginalmente superior ao percentil 95. Para explorar esta possibilidade, foi realizada outra AFE com 3 fatores, com extração de MV e rotação oblíqua. No entanto, apenas um item (item 4) saturou um dos fatores, sendo, no entanto, insuficiente para representar um fator distintivo. Além disso, o facto de o scree plot (cf. Fig. 1) indicar que o ponto de inflexão na inclinação está no segundo fator, reforçou o número de fatores a extrair (Cattell, 1966). Assim, foram considerados dois fatores, identificados como estático e dinâmico. 
Quadro 3. Índices de ajustamento da Análise Fatorial Confirmatória e Análise Fatorial Confirmatória Multigrupo

\begin{tabular}{|c|c|c|c|c|c|c|c|c|c|}
\hline & $\chi^{2}$ & df & $\chi^{2 / d f}$ & CFI & TLI & SRMR & RMSEA $[90 \% \mathrm{CI}]$ & $\Delta \mathrm{CFI}$ & $\triangle \mathrm{RMSEA}$ \\
\hline $\begin{array}{l}\text { Análise Fatorial } \\
\text { Confirmatória }\end{array}$ & & & & & & & & & \\
\hline $\begin{array}{l}2 \text { Fatores } \\
\text { (erros do } \\
\text { item } 6 \text { e } 10 \\
\text { correlacionados) }\end{array}$ & 106.95 & 52 & 2.06 & .97 & .96 & .035 & $\begin{array}{c}.061 \\
{[.044, .077]}\end{array}$ & - & - \\
\hline $\begin{array}{l}\text { Análise Fatorial } \\
\text { Confirmatória } \\
\text { Multigrupo }\end{array}$ & & & & & & & & & \\
\hline $\begin{array}{l}\text { Invariância } \\
\text { Configuracional }\end{array}$ & 175.89 & 106 & 1.66 & .96 & .95 & .049 & $\begin{array}{c}.048 \\
{[.035, .061]}\end{array}$ & - & - \\
\hline $\begin{array}{l}\text { Invariância } \\
\text { Métrica }\end{array}$ & 190.58 & 116 & 1.64 & .96 & .95 & .053 & $\begin{array}{c}.048 \\
{[.035, .059]}\end{array}$ & -.003 & .000 \\
\hline $\begin{array}{l}\text { Invariância } \\
\text { Escalar }\end{array}$ & 196.86 & 128 & 1.54 & .96 & .96 & .053 & $\begin{array}{c}.044 \\
{[.031, .055]}\end{array}$ & .001 & .004 \\
\hline
\end{tabular}

\section{Análise Fatorial Confirmatória (AFC)}

A AFC usando a estimativa de MV ( $\mathrm{n}_{\text {subgrupo }}$ 2=286) foi realizada para avaliar o ajustamento do modelo aos dados. O modelo bifatorial previamente identificado pela AFE foi examinado, obtendo os seguintes índices de ajustamento: $\chi^{2}$ $=128.89, d f=53, \chi^{2} / d f=2.43, \mathrm{CFI}=.96$, TLI=.95, $\mathrm{RMSEA}=.061$ e $\mathrm{SRMR}=.041$. Embora globalmente o modelo tenha apresentado um bom ajustamento aos dados, confirmando os resultados do modelo bifatorial EFA, a revisão dos índices de modificação sugeriu a correlação dos erros dos itens 6 e 10. Assim, um modelo subsequente com os erros correlacionados apresentou um melhor ajustamento aos dados: $\chi^{2}=106.95, d f=52, \chi^{2}$ / $d f=2.06, \quad$ CFI $=.97, \quad$ TLI $=.96, \quad$ RMSEA $=.061$ e SRMR=.035 (cf. Quadro 3). Os resultados demonstraram que o modelo de dois fatores da TIIE ofereceu um melhor ajustamento aos dados, e todos os índices considerados alcançaram os critérios mínimos aceitáveis. As cargas fatoriais padronizadas de todos os itens estavam acima de .60. Os dois fatores apresentaram uma correlação de $r=-.79$ (cf. Fig. 2).

\section{Invariância de Medida - Género}

A AFC multigrupo foi realizada para verificar se a estrutura fatorial da escala TIIE seria igual para ambos os géneros. Para avaliar a invariância configuracional, não foram incluídas restrições no modelo, e os resultados confirmaram que este modelo revelava um bom ajustamento aos dados (Quadro 3, $\chi^{2} \quad(106)=175.89, \chi^{2} / \mathrm{df}=1.66, \mathrm{CFI}=$ $.96, \mathrm{TLI}=.95$, SRMR $=.049$, RMSEA $=.048[90 \%$
$\mathrm{IC}=.035, .061]) . \quad$ Em seguida, o modelo de invariância métrica, que restringe as cargas fatoriais e as correlações a serem iguais entre grupos, foi explorado. Os resultados revelaram um bom ajustamento ao modelo, confirmando a invariância métrica quanto ao género (cf. Quadro 3, $\chi^{2} \quad(116)=190.58, \quad \chi^{2} / \mathrm{df}=1.64, \quad \mathrm{CFI}=.96$, TLI $=.95, \quad$ SRMR $=.053, \quad$ RMSEA $=.048 \quad[90 \%$ $\mathrm{IC}=.035, .059])$. Por fim, o modelo de invariância escalar foi testado, restringindo-se os interceptos das variáveis medidas a serem iguais entre géneros, além das cargas fatoriais das variáveis latentes. $\mathrm{O}$ bom ajustamento dos dados ao modelo mais restrito confirmou a invariância escalar para os grupos (cf. Quadro 3, $\chi^{2}(128)=196.86, \chi^{2} / \mathrm{df}$ $=1.54, \quad$ CFI $=.96, \quad$ TLI $=.96, \quad$ SRMR $=.053$, RMSEA=.044 [90\% IC=.031, .055]). As mudanças no CFI e RMSEA foram também consideradas e não excederam o valor máximo de .01 e .015, respetivamente (cf. Quadro 3).

\section{Consistência Interna}

A consistência interna da escala foi avaliada através do alfa de Cronbach. Tanto a escala total $(\alpha=.91)$ como as duas subescalas (estática $\alpha=.88 \mathrm{e}$ dinâmica $\alpha=.86$ ) apresentaram boa consistência interna. O coeficiente de split-half da escala TIIE foi de .90. A correlação item-escala corrigida variou entre .59 e .75 na escala total, de .69 a .77 na subescala estática e de .61 a .72 para a subescala dinâmica, superando, assim, o valor mínimo satisfatório de .30 (Nunnally \& Bernstein, 1994). Globalmente, os diferentes indicadores refletiram a boa consistência interna da TIIE. 
Quadro 4. Correlações de Pearson entre as TIIE e as medidas de TII e Emoções face à escola

\begin{tabular}{|c|c|c|c|c|}
\hline & \multirow{2}{*}{$\begin{array}{c}\text { Análises Descritivas } \\
\qquad M(D P)\end{array}$} & \multicolumn{3}{|c|}{ Correlação } \\
\hline & & 1 & 2 & 3 \\
\hline 1. TIIE & $55.11(9.49)$ & 1 & & \\
\hline 2. TIIE estático & $15.08(5.70)$ & $-.93 * *$ & 1 & \\
\hline 3. TIIE dinâmico & $28.18(4.69)$ & $.90 * *$ & $-.67 * *$ & 1 \\
\hline 4. TII & $57.24(9.03)$ & $.36 * *$ & $-.33 * *$ & $.32 * *$ \\
\hline 5. TII estático & $13.89(5.55)$ & $-.35 * *$ & $.39 * *$ & $-.24 * *$ \\
\hline 6. TII dinâmico & $29.04(4.65)$ & $.30 * *$ & $-.21 * *$ & $.36 * *$ \\
\hline 7. Emoções Escola & $33.42(5.88)$ & $.21 * *$ & $-.18 * *$ & $.21 * *$ \\
\hline 8. Emoções Positivas Escola & $14.87(3.46)$ & $.21 * *$ & $-.16 * *$ & $.22 * *$ \\
\hline 9. Emoções Negativas Escola & $16.46(3.86)$ & $-.13 * *$ & $.13^{* *}$ & $-.12 * *$ \\
\hline
\end{tabular}

Nota. TIIE=Teorias Implícitas de Inteligência Emocional; TII=Teorias Implícitas de Inteligência; Emoções Escola=Emoções dos alunos face à Escola; $\mathrm{n}=575$.

$* * p<.001 ; * p<.05$.

\section{Validade Discriminante}

Com o objetivo de explorar a validade discriminante da escala, foi explorado através da correlação de Pearson, o grau de associação da escala de TIIE com a escala de Teorias Implícitas de Inteligência (TII) e ainda com a escala de Emoções dos Alunos face à Escola. Os resultados evidenciaram correlações significativas entre todas as variáveis: correlações de magnitude forte entre a TIIE e respetivas subescalas, de nível moderado entre as subescalas da TIIE e da TII e de nível baixo entre a TIIE e a de Emoções dos Alunos face à escola (cf. Quadro 4).

\section{Discussão}

A literatura sobre as teorias implícitas tem demonstrado o impacto destas no comportamento e no desempenho dos indivíduos em diversos contextos de existência (Burnette et al., 2013; Costa \& Faria, 2018). No entanto, a investigação que explora o impacto das teorias implícitas da inteligência emocional é quase inexistente, sobretudo devido à falta de medidas para avaliar e, consequentemente, aprofundar a compreensão do constructo. O objetivo deste estudo foi, portanto, desenvolver e estabelecer as qualidades psicométricas de uma escala de Teorias Implícitas de Inteligência Emocional (TIIE).

Globalmente, a análise fatorial exploratória sugeriu a estrutura bifatorial da TIIE - um fator representativo da teoria estática e outro da dinâmica da IE. Embora a análise fatorial paralela sugerisse a presença de um terceiro fator ligeiramente acima do percentil 95 - foram valorizados os outros critérios disponíveis, uma vez que a análise paralela pode ser um critério falível quando os valores próprios dos fatores estão próximos do ponto de corte do percentil 95 (Ruscio \& Roche, 2012). Além disso, o modelo fatorial exploratório alternativo, com 3 fatores, teria um fator representado apenas por um item, o que não constituiria uma dimensão psicometricamente sólida. Mais ainda, a estrutura com dois fatores foi consistente com a estrutura teórica esperada e, portanto, assumida nas análises seguintes.

$\mathrm{Na}$ AFC, o modelo de dois fatores revelou excelentes índices de ajustamento global e relativo, confirmando a estrutura estabelecida. A estrutura latente - estática e dinâmica - é consistente com outros estudos de teorias implícitas (De Castella \& Byrne, 2015; Dupeyrat \& Mariné, 2005), fornecendo evidência empírica que suporta a dimensionalidade teórica do constructo. $\mathrm{O}$ fator estático revelou melhores índices psicométricos do que o fator dinâmico, explicando maior percentagem de variância total na AFE e, em média, apresentando melhores pesos fatoriais na AFC.

A análise dos itens indicou que, ainda que não a um nível problemático, os itens 6,10 e 12 podem vir a ser refinados no âmbito de uma análise mais aprofundada. Embora estes itens apresentem indicadores psicométricas mais baixos (por exemplo, comunalidades, cargas fatoriais), não será recomendável a sua exclusão, até que novos estudos confirmem a sua validade psicométrica.

A invariância de medida de TIIE quanto ao género foi avaliada segundo níveis de invariância 
consecutivamente mais restritivos. Neste estudo foi estabelecida a invariância configuracional, métrica e escalar da TIIE entre os grupos de género. Com base nestes resultados, a escala TIIE pode ser usada em ambos os grupos, uma vez que mede o constructo na mesma proporção, os itens são interpretados de maneira similar e a escala de respostas está a ser adotada da mesma forma por rapazes e raparigas.

Em relação à fiabilidade da escala, verificouse que os scores da escala total e das subescalas da TIIE demonstraram bons níveis de fiabilidade $(\alpha$ acima de .86; coeficiente de Split-half de .90).

Além disso, verificou-se uma correlação negativa moderada entre as duas subescalas da TIIE $(r=-.67)$. A correlação moderada indica a associação entre as duas dimensões, como esperado, já que ambas partilham a avaliação do mesmo constructo, embora a correlação negativa informe sobre as perspetivas opostas no mesmo contínuo.

Finalmente, explorou-se a validade discriminante da escala de TIIE, correlacionandoa com outra medida de teorias implícitas, no caso, de inteligência (TII). Os resultados evidenciaram a correlação moderada entre TIIE e TII (escala total e subescalas), confirmando a associação parcial das duas medidas de teorias implícitas, mas destacando a especificidade da avaliação de cada constructo - inteligência emocional versus inteligência. Assim, os resultados confirmaram que estas medidas avaliam constructos relacionados, mas distintos. As dimensões estáticas da TIIE e da TII correlacionaram-se inversamente, tanto com as dimensões dinâmicas quanto com as escalas totais das teorias implícitas. Estes resultados são congruentes com as evidências empíricas e com o esperado teoricamente, uma vez que a perspetiva estática e dinâmica correspondem a polos opostos do mesmo contínuo (De Castella \& Byrne, 2015; Dupeyrat \& Mariné, 2005). A TIIE estava também significativamente correlacionada com a medida das emoções dos alunos face à escola. Os resultados destacaram a correlação positiva entre a subescala dinâmica do TIIE e as emoções positivas dos alunos face à escola, de acordo com o previsto, uma vez que estudos anteriores indicaram que os indivíduos com conceções mais dinâmicas tendem a experimentar menos emoções negativas (Kappes \& Schikowski, 2013). O facto de os indivíduos dinâmicos adotarem estratégias emocionais mais adaptativas (Kappes \& Schikowski 2013; Tamir et al., 2007), pode leválos a regular mais facilmente o seu humor, no contexto académico, passando mais facilmente de um estado emocional negativo para um estado emocional mais positivo e agradável.

O presente estudo não está ausente de limitações. Primeiramente, o desenho transversal limitou as análises da validade temporal da escala. Portanto, a validade teste-reteste e a invariância temporal não puderam ser exploradas. Mais ainda, este estudo incluiu apenas alunos do $100^{\circ}$ ano, de modo que a adequação da TIIE a alunos mais velhos do ensino secundário não pôde ser testada. Por outro lado, outras importantes variáveis critério poderiam ter sido exploradas (por exemplo, a IE dos alunos - capacidade e traço - e indicadores objetivos e subjetivos de desempenho académico). Assim, os resultados preliminares desta medida devem ser aprofundados, confirmados e validados em diferentes amostras, em particular, em outros níveis de ensino, faixas etárias e contextos culturais.

Em conclusão, o presente estudo constituiu uma oportunidade para aprofundar a compreensão das teorias implícitas da inteligência emocional, oferecendo evidências empíricas da fiabilidade, validade e utilidade da TIIE no contexto do ensino secundário.

\section{Referências}

Billings, C. W. S., Downey, L. A., Lomas, J. E., Lloyd, J., \& Stough, C. (2014). Emotional intelligence and scholastic achievement in pre-adolescent children. Personality and Individual Differences, 65, 14-18. doi:10.1016/j.paid.2014.01.017

Blackwell, L. S., Trzesniewski, K. H., \& Dweck, C. S. (2007). Implicit theories of intelligence predict achievement across an adolescent transition: A longitudinal study and an intervention. Child Development, 78, 246-263. doi:10.1111/j.1467-8624.2007.00995.x

Brackett, M., Rivers, S. E., \& Salovey, P. (2011). Emotional intelligence: Implications for personal, social, academic, and workplace 
success. Social and Personality Psychology Compass, 5, 88-103. doi:10.1111/j. 4541751-9004.2010.00334.x.

Burnette, J. L., O'Boyle, E. H., VanEpps, E. M., Pollack, J. M., \& Finkel, E. J. (2013). Mindsets matter: A meta-analytic review of implicit theories and self-regulation. Psychological Bulletin, 139, 655-701. doi:10.1037/a0029531

Cabello, R., \& Fernández-Berrocal, P. (2015). Implicit theories and ability emotional intelligence. Frontiers in Psychology, 6(700), 1-8. doi:10.3389/fpsyg.2015.00700

Cattell, R. B. (1966). The scree test for the number of factors. Multivariate Behavioral Research, 1, 245-276.

Chen, F. F. (2007). Sensitivity of goodness of fit indexes to lack of measurement invariance. Structural Equation Modeling, 14, 464-504. doi: 10.1080/10705510701301834

Chiu, C., Hong, Y., \& Dweck, C. S. (1997). Lay dispositionism and implicit theories of personality. Journal of Personality and Social Psychology, 73, 19-30.

doi:10.1037/0022-3514.73.1.19

Costa, A., \& Faria, L. (2015). The impact of emotional intelligence on academic achievement: A longitudinal study in Portuguese secondary school. Learning and Individual Differences, 37, 38-47. doi:10.1016/j.lindif.2014.11.011

Costa, A., \& Faria, L. (2018). Implicit theories of intelligence and academic achievement: A meta-analytic review. Frontiers in Psychology. 9, 829. doi:10.3389/fpsyg.2018.00829

De Castella, K., \& Byrne, D. (2015). My intelligence may be more malleable than yours: The revised implicit theories of intelligence (self-theory) scale is a better predictor of achievement, motivation, and student disengagement. European Journal of Psychology of Education, 30, 245-267. doi:10.1007/s10212-015-0244-y

De Castella, K., Goldin, P., Jazaieri, H., Ziv M., Dweck, C. S., \& Gross, J. J. (2013). Beliefs about emotion: Links to emotion regulation, well-being, and psychological distress. Basic and Applied Social Psychology, 35, 497-505. 10.1080/01973533.2013.840632

Dupeyrat, C., \& Mariné, C. (2005). Implicit theories of intelligence, goal orientation, cognitive engagement, and achievement: A test of Dweck's model with returning to school adults. Contemporary Educational Psychology. 30, 43-59.

doi:10.1016/j.cedpsych.2004.01.007

Dweck, C. S. (1999). Self-Theories: Their role in motivation, personality, and development. Philadelphia, PA: Psychology Press.

Dweck, C. S. (2012). Implicit theories. In P. Van Lange, A. Kruglanski, \& T. Higgins (Eds.), The handbook of theories of social psychology (pp. 43-62). London: SAGE Publications Ltd.

Dweck, C. S., \& Leggett, E. L. (1988). A socialcognitive approach to motivation and personality. Psychological Review, 95, 256273. doi:10.1037/0033-295X.95.2.256

Faria, L. (2008). Motivação para a competência: $O$ papel das concepções pessoais de inteligência no desempenho e no sucesso. Porto: Livpsic.

Fernández-Berrocal, P., \& Extremera, N. (2006). Emotional intelligence: A theoretical and empirical review of its first 15 years of history. Psicothema, 18(1), 7-12.

Hu, L., \& Bentler, P. M. (1999). Cutoff criteria for fit indexes in covariance structure analysis: Conventional criteria versus new alternatives. Structure Equation Modeling, 6, 1-55.

Kappes, A., \& Schikowski, A. (2013). Implicit theories of emotion shape regulation of negative affect. Cognition and Emotion, 27(3), 1-9.

Mavroveli, S., \& Sánchez-Ruiz, M. J. (2011). Trait emotional intelligence influences on academic achievement and school behaviour. British Journal of Educational Psychology, 81(1), 112-134.

Mayer, J. D., Roberts, R. D., \& Barsade, S. G. (2008). Human abilities: Emotional intelligence. Annual Review of Psychology, 59, 507-536.

Mayer, J. D., \& Salovey, P. (1997). What is emotional intelligence? In P. Salovey, \& D. Sluyter (Eds.), Emotional development and emotional intelligence: Educational applications (pp. 3-31). New York: Basic Books.

Mestre, J., Guil, R., Lopes, P., Salovey, P., \& GilOlarte, P (2006). Emotional intelligence and 
social and academic adaptation to school. Psicothema, 18(1), 112-117.

Nunnally, J. C., \& Bernstein, I. H. (1994). Psychometric theory. New York: McGraw Hill.

O'Connor, B.P. (2000). SPSS and SAS programs for determining the number of components using parallel analysis and Velicer's MAP test. Behavior Research Methods, Instruments, \& Computers, 32(3), 396-402. doi:10.3758/BF03200807

Pekrun, R., Goetz, T., Frenzel, A. C., Barchfeld, P., \& Perry, R. P. (2011). Measuring emotions in students' learning and performance: The Achievement Emotions Questionnaire (AEQ). Contemporary Educational Psychology, 36, 36-48. doi:10.1016/j.cedpsych.2010.10.002

Pepi, A., Faria, L., \& Alesi, M. (2006). Personal conceptions of intelligence, self-esteem, and school achievement in Italian and Portuguese students. Adolescence, 41, 615-631.

Petrides, K. V., Pita, R., \& Kokkinaki, F. (2007). The location of Trait Emotional Intelligence in personality factor space. British Journal of Psychology, 98, 273-289. doi:10.1348/000712606X120618

Romero, C., Master, A., Paunesku, D., Dweck, C. S., \& Gross, J. (2014). Academic and emotional functioning in middle school: The role of implicit theories. Emotion, 14, 227234. doi:10.1037/a0035490

Ruscio, J., \& Roche, B. (2012). Determining the number of factors to retain in an exploratory factor analysis using comparison data of known factorial structure. Psychological Assessment, 24(2), 282-292. doi: $10.1037 / \mathrm{a} 0025697$

Salessi, S., \& Omar, A. (2016). Desarollo y validación de una escala para medir actuación emocional en el trabajo. Revista Iberoamericana de Diagnóstico y Evaluación - e Avaliação Psicológica, 41(1), 66-79.

Salovey, P., \& Mayer, J.D. (1990). Emotional intelligence. Imagination, Cognition and Personality, 9, 185-21.

Schumann, K., Zaki, J., \& Dweck, C. S. (2014). Addressing the empathy deficit: Beliefs about the malleability of empathy predict effortful responses when empathy is challenging. Journal of Personality and Social
Psychology, 107,

475-493.

doi: 10.1037/a0036738

Tamir, M., John, O. P., Srivastava, S., \& Gross, J. J. (2007). Implicit theories of emotion: Affective and social outcomes across a major life transition. Journal of Personality and Social Psychology, 92, 731-744. doi:10.1037/0022-3514.92.4.731

Teixeira, F., \& Araújo, A. (2018). Psychometric properties of the Mayer-Salovey-Caruso Emotional Intelligence Test - MSCEIT V2.0: A Systematic review of the literature. Revista Iberoamericana de Diagnóstico y Evaluación - e Avaliação Psicológica, 48(3), 163-176.

Van Rooy, D. L., \& Viswesvaran, C. (2004). Emotional intelligence: A meta-analytic investigation of predictive validity and nomological net. Journal of Vocational Behavior, 65, 71-95. doi:10.1016/S0001-8791(03)00076-9. 